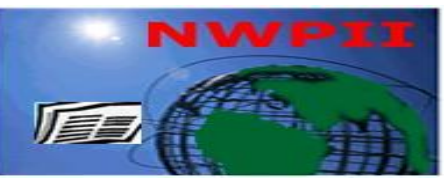

American Journal of Biomedical Sciences

ISSN: 1937-9080

nwpii.com/ajbms

\title{
Evaluation of Analgesic, Anti-inflammatory and CNS Depressant Potential of Dendrophthoe falcata (Linn.) Leaves Extracts in Experimental Mice Model
}

\author{
Anamul Haque*', Afrina Zaman², Tahmina², Motaher Hossain ${ }^{3}$, Icha Sarker ${ }^{3}$, Saiful Islam ${ }^{3}$, \\ Abdullah Al Baki ${ }^{2}$ \\ ${ }^{1}$ Department of Pharmacy, Comilla University, Kotbari, Comilla, Bangladesh \\ ${ }^{2}$ Department of Pharmacy, Southeast University, Banani, Dhaka, Bangladesh \\ ${ }^{3}$ Department of Pharmacy, Rajshahi University, Rajshahi, Bangladesh \\ *Corresponding Author \\ Anamul Haque \\ Lecturer \\ Department of Pharmacy \\ Comilla University \\ Kotbari, Comilla \\ Bangladesh \\ Mob: +088-01912894766 \\ Email: pharmaripon@gmail.com
}

Received: 20 May 2014; | Revised: 5 September 2014; | Accepted: 18 September 2014

\begin{abstract}
Plants are extraordinary reservoir of bioactive natural products, many of which exhibit chemical and structural features on outstanding biological activities in animals. Dendrophthoe falcata (Linn.) is an important parasitic plant extensively used in folk medicine. In this regard, the aim of this study was to investigate the possible analgesic, anti-inflammatory and central nervous system (CNS) depressant activities of ethanol, aqueous, chloroform and petroleum ether extracts of D. falcata (Linn.) leaves (AEDFL, EEDFL, CEDFL and PEDFL) on Swiss albino mice. The analgesic activity was determined by tail immersion and acetic acid induced writhing methods at a dose of 50, 100 and $200 \mathrm{mg} / \mathrm{kg}$, p.o. using morphine sulfate as a standard drug at a dose of $5 \mathrm{mg} / \mathrm{kg}$ of body weight. Carrageenan-induced paw edema model was applied to find out anti-inflammatory potential at the same doses of analgesic activity. Here ibuprofen was treated as a standard drug at $10 \mathrm{mg} / \mathrm{kg}$ body weight. The CNS depressant effect of the extracts were evaluated by using open field and hole cross tests. All the extracts exhibited significant $\left(\mathrm{P}^{\mathrm{b}}<0.01, \mathrm{P}^{\mathrm{a}}<0.001\right)$ analgesic, antiinflammatory and CNS depressant effects at dose dependant manner. Petroleum ether extracts (PEDFL) showed maximum analgesic effect (77.45\% inhibition of abdominal writhing and $82.12 \%$ elongation of tail withdrawal time) at $200 \mathrm{mg} / \mathrm{kg}$ dose. Moreover, the highest anti-inflammatory potential $(90.24 \%$ inhibition of paw edema volume) was showed by petroleum ether extract at $200 \mathrm{mg} / \mathrm{kg}$ dose. On the other hand, ethanol extract (EEDFL) proved to have significant $(97.33 \%$ and $85.91 \%$ inhibition of locomotion in open field and
\end{abstract}


in hole cross test respectively) CNS-depressant activities after $120 \mathrm{~min}$ of oral administration of the extract at $200 \mathrm{mg} / \mathrm{kg}$ dose.

Keywords: Dendrophthoe falcata, Analgesic, Anti-inflammatory, CNS depressant.

\section{Introduction}

Dendrophthoe falcata (Linn.) belongs to the family of Loranthaceace and is commonly known as 'Vanda' in the Indian Ayurvedic System of Medicine $[1,2]$. It is a perennial climbing woody hemiparasitic [3], and evergreen shrub that is frequently observed on different host plants such as Mangifera indica, Melia azadirecta, Psidium guayava etc. Bark of D. falcata is grey. Its leaves are thick, coriaceous, much variable in shape usually opposite 7.5 to 18 by $2-10 \mathrm{~cm}$. Flower of the plant are stout, unilateral racemes often two from an axil pedicle. These are ovate sub-acute, concave and scarlet or orange or less commonly pink or white in color. Anthers are linear, equal in length to the free portion of the filament. Berries of D. falcata are 8-13 mm long ovoid oblong, pink, smooth crowned by a cup- shaped calyx. $D$. falcata comprises of 20 species where about 7 species are found in Indian subcontinent [4], that are indigenous to tropical and sub-tropical regions especially in India, Sirilanka, China, Australia, Bangladesh, Malayasia, Myanmar, Thailand and Indo-china [2,3].

Entire plant is used extensively in traditional system of medicine as an aphrodisiac, astringent, narcotic and diuretic. It is applied for the treatment of pulmonary tuberculosis, asthma, menstrual disorders, swellings, wounds, ulcers, strangury and psychic disorders [3]. Leaf paste is used in skin diseases. Its paste is applied on boils setting, dislocated bones and extracting pus [2]. It is also used in renal and vesical calculi and vitiated conditions of kapha and pitta. D. falcata is reported to have antilithiatic, cytotoxic, immunomodulatory, antimicrobial, antioxidant and hepatoprotective activity. In the traditional system of medicine D. falcata is recommended for the treatment of epilepsy and paralysis [1]. Also, it has antibiofilm and anti-quorum effects against a wide variety of microorganisms [5]. The plant is also proved to have anti-fertility efficacy in Wistar female rats [6,7], as well as anticancer effect against human breast cancer $[4, \underline{8}]$.

Preliminary phytochemical screening revealed the presence of carbohydrates, phytosterols, flavonoids, glycosides and phenolic compounds [3]. It is reported to contain biologically active substances such as quercetin, tannins, $\beta$-sitosterol, $\beta$-amyrin, oleanolic acid [4]. Several active chemical constituents such as stigmasterol, kaempferol, quercetin-3-Orhamnoside, rutin, and myricetin and their glycosides, (+)-catechin, leucocyanidin, gallic acid, chebulinic acid and some pentacyclic triterpenes: rhamnopyranoside and quercetin-3-O- $\alpha$-Lrhamnopyranoside, etc are isolated and identified in it (D. falcata) [2]. Moreover, three new pentacyclc triterpenes such as -(3ß-acetoxy-1ß(2-hydroxy-2-propoxy)-11 $\alpha$-hydroxy-olean-12ene, 3ß-acetoxy-11 $\alpha$-ethoxy-1ß-hydroxy-olean-

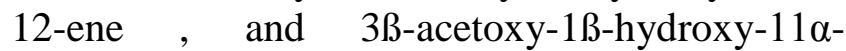
methoxy-0lean-12-ene have been reported in the plant $[9,10]$.

Inflammation is a crucial and necessary function of the innate immune system, protecting the host against pathogens and initiating a specific immune response. Hence, inflammation is a complex process that is frequently associated with pain [11]. Anxiety is being explained as a psychological and physiological state marked by cognitive, somatic, emotional and behavioral elements. Together, these components provoke a disagreeable emotion associated with fear, worry as well as restlessness. Therefore, it can be an obstacle in everyday life [12]. It is proved that prolong use of synthetic drugs of all classes are not only harmful but also very expensive to develop. So, the associated complexities of synthetic drugs have lead to a shift towards locating natural resources showing therapeutic potentials [13]. These conventional medicines are associated with unpleasant side effects or toxic effect such as gastrointestinal disturbances [14], and have shown only limited success against 
chronic diseases [15]. Drugs are increasingly being developed from natural origins, offering a very promising approach to identify novel therapeutic agents [16]. Natural products have long been used in traditional medicine to treat inflammation and other inflammation-related diseases, and the raw materials of these products are often used to develop new drugs $[17,18]$.

The present study was undertaken to investigate analgesic, anti-inflammatory and CNS depressant activities of aqueous, ethanol, chloroform and petroleum ether extracts of $D$. falcata leaves in Swiss albino mice that may unveil the rationality of use of the plant as traditional medicines and potentiality of it in the herbal medicine.

\section{Materials and methods}

\subsection{Plant materials}

For the investigation, Dendrophthoe falcata (Linn.) leaves, mistletoe of Swietenia fabrilis tree were collected from Joypurhat, Bangladesh in September, 2012 and identified by experts of the Bangladesh National Herbarium, Dhaka, where a voucher specimen has also been retained with accession no. 39432. The collected leaves were cleaned and dried for one week in electric oven, and pulverized into a coarse powder using a suitable grinder. The powder was stored in an airtight container and kept in a cool, dark, and dry place until further analysis.

\subsection{Extract preparation}

Approximately, $800 \mathrm{~g}$ of powdered material was placed in a clean, flat-bottomed glass container and soaked in ethanol and similarly $400 \mathrm{~g}$ of the powder was soaked in distilled water. Both of the containers with their contents were sealed and kept for 5 days. Then the extraction was carried out by using an Ultrasonic Sound Bath accompanied by sonication (40 minutes). The entire mixture, then, underwent a coarse filtration by a piece of clean and white cotton material. Then the extracts were filtered through Whitman filter paper (Bibby RE200, Sterilin Ltd., UK) and were concentrated to obtain the ethanol $(12 \mathrm{~g})$ and aqueous (4g) extracts. Ethanol extract was divided into two portions. One portion $(2 \mathrm{~g})$ was poured into a glass vial to be tested as crude ethanol extract, whereas the second portion $(10 \mathrm{~g})$ was dissolved in $100 \mathrm{~mL}$ of ethanol, and partitioned successively with chloroform and petroleum ether. The fractions were then concentrated using a rotary evaporator to obtain chloroform fraction (yield weight $1.5 \mathrm{~g}$ ), and petroleum ether fraction (yield weight $2.60 \mathrm{~g}$ ). This process rendered a gummy concentrated reddish black color. The gummy extracts were transferred to a closed container for further use and storage.

\subsection{Drugs and chemicals}

Ethanol, chloroform and petroleum ether were used as solvent for the extraction of the leaves and acetic acid was used in writhing test. These chemicals were purchased from Merck, Germany. Tween-80 was also collected from Merck, Germany. Ibuprofen, diazepam and morphine sulfate were collected from Square Pharmaceuticals Ltd. Bangladesh. All the chemicals used in these investigations were of analytical reagent grade.

\subsection{Animals}

Swiss albino mice of either sex weighing approximately $25-30 \mathrm{~g}$ were used for these experiments. These mice were purchased from the animal research branch of International Centre for Diarrhoeal Disease and Research, Bangladesh (ICDDR, B). After their purchase, the mice were kept in standard environmental conditions $\left(25.0 \quad \pm 2.0^{\circ} \mathrm{C} \& 55-65 \%\right.$ relative humidity and $12 \mathrm{~h} \mathrm{light/dark} \mathrm{cycle)} \mathrm{for} \mathrm{four}$ weeks to acclimate and fed ICDDR, B formulated rodent food and water ad libitum. The experimental procedures involving animals were conducted in accordance with the guidelines of Southeast University, Dhaka, Bangladesh. The study protocol was approved by Institutional Animal, Medical Ethics, Biosafety and Biosecurity Committee of the University. The set of rules followed for animal experiment were approved by the institutional animal ethical committee and handled in accordance with international guidelines for care and use of laboratory animals [19]. 


\subsection{Analgesic activity}

\subsubsection{Acetic acid-induced writhing test}

The analgesic activity of the extracts (AEDFL, EEDFL, CEDFL and PEDFL) was studied by using acetic acid-induced writhing model in Swiss albino mice, described by Koster R. et al. [20]. The animals were divided into fourteen groups with six mice in each group. Group I animals received vehicle (1\% tween 80 in water, p.o.), animals of group II received morphine sulfate at $5 \mathrm{mg} / \mathrm{kg}$ body weight while animals of groups III-XIV received extracts of the leaves. Group III, IV and V received AEDFL at a dose of $50,100,200 \mathrm{mg} / \mathrm{kg}$ body weight (p.o.). Group VI, VII and VIII received EEDFL at a dose of $50,100,200 \mathrm{mg} / \mathrm{kg}$ body weight (p.o.). Group IX, X and XI received CEDFL at a dose of 50,100, $200 \mathrm{mg} / \mathrm{kg}$ body weight (p.o.). Group XII, XIII and XIV received PEDFL at a dose of 50,100, $200 \mathrm{mg} / \mathrm{kg}$ body weight (p.o.). After 30 minutes of vehicle, standard drug and extracts administration, $0.6 \% \mathrm{v} / \mathrm{v}$ acetic acid was administered into the peritoneum of each animal. The writhing response, which consists of a contraction of the abdominal muscle together with a stretching of the hind limbs, was determined for 20 minutes after a latency period of 5 minutes. The Percentage reduction of abdominal constriction indicates the percentage protection against it which was taken as an index of analgesia. It was calculated as:

\% Inhibition $=\left[\left(\mathrm{N}_{\mathrm{c}}-\mathrm{N}_{\mathrm{t}}\right) / \mathrm{N}_{\mathrm{c}}\right] \times 100$

Where, $\mathrm{N}_{\mathrm{c}}=$ number of writhing of the control group, $\mathrm{N}_{\mathrm{t}}=$ number of writhing of the treated group

\subsubsection{Tail immersion test}

Tail immersion test was done by the method described by Toma W. et al. [21]. This test is based on the observation that morphine like drugs selectively prolongs the reaction time of the typical tail-withdrawal reflex in mice. According to the method, vehicle ( $1 \%$ tween 80 in water, p.o.), morphine sulfate $(5 \mathrm{mg} / \mathrm{kg}$, p.o.), AEDFL (50, 100 and $200 \mathrm{mg} / \mathrm{kg}$, p.o.), EEDFL (50, 100 and $200 \mathrm{mg} / \mathrm{kg}$, p.o.), CEDFL (50, 100 and 200 $\mathrm{mg} / \mathrm{kg}$, p.o.) and PEDFL (50, 100 and 200 $\mathrm{mg} / \mathrm{kg}$, p.o.) were administered to the mice of respective groups $30 \mathrm{~min}$ before the experiment. About $3 \mathrm{~cm}$ tail of each mouse was immersed in warm water kept constant at $55 \pm 1{ }^{\circ} \mathrm{C}$. The reaction time was the time taken by the mouse to deflect the tail. The latency period of the tailwithdrawal response was taken as the index of analgesia and was determined before drug treatment and at 30,60, 90, and 120 min after oral administration of the extracts with respective dose. To determine the baseline, each animal was tested before administration of drug/extracts. A cut off period of $10 \mathrm{~s}$ was maintained to avoid tail tissue damage. The results of the tail immersion test are expressed as a percentage of the maximal possible effect (\%MPE), which was calculated using the following formula:

$\% \mathrm{MPE}=[($ Post drug latency - pre drug latency $) /$ (Cut off period-pre drug latency) $] \times 100$

\subsection{Anti-inflammatory activity}

\subsubsection{Carrageenan induced paw edema test}

Anti-inflammatory activity of the extracts was evaluated by carrageenan-induced paw edema model [22]. Swiss albino mice (25-30g) of both sexes were divided into fourteen groups of six animals in each. The test groups received 50, 100 and $200 \mathrm{mg} / \mathrm{kg}$ body weight p.o. of the extract AEDFL, EEDFL, CEDFL and PEDFL. The reference group received ibuprofen (10 $\mathrm{mg} / \mathrm{kg}$ p.o.) body weight while the control group received vehicle (1\% tween 80 in water, p.o.). After 30 min, 100 $\mu 11 \%$ carrageenan suspension in normal saline was injected into the sub-plantar tissue of the left hind paw of each animal. The paw volume was measured before and after carrageenan injection at 1,2,3 and 4 hrs using a Plethysmometer 7150 (Ugo Basile, Italy). The extant of reduction of paw volume revealed the ability of inflammation reduction. The percentage inhibition of inflammation was calculated by the following formula:

$\%$ Inhibition $=\left[\left(\mathrm{V}_{0}-\mathrm{V}_{\mathrm{S}}\right) / \mathrm{V}_{0}\right] \times 100$ 
Where, $\mathrm{V}_{0}$ is the average paw edema volume of the control group, $\mathrm{V}_{\mathrm{S}}$ is average paw edema volume of the treatment and standard group.

\subsection{CNS depression activity}

\subsubsection{Open field test}

The effect of the aqueous, ethanol, chloroform and petroleum ether extracts on the spontaneous locomotor activity of the experimental animals was evaluated by the method, described by Gupta et al. [23]. Eighty four albino mice were divided into fourteen groups $(n=6)$. Vehicle ( $1 \%$ tween 80 in water, p.o.), diazepam (2 mg/kg, p.o.), AEDFL (50, 100 and $200 \mathrm{mg} / \mathrm{kg}$, p.o.), EEDFL (50, 100 and 200 $\mathrm{mg} / \mathrm{kg}$, p.o.), CEDFL (50, 100 and $200 \mathrm{mg} / \mathrm{kg}$, p.o.) and PEDFL (50, 100 and $200 \mathrm{mg} / \mathrm{kg}$, p.o.) were administered to different groups (I- XIV) before 30minutes of experiment. The animals were placed on the floor of an open field (100 $\mathrm{cm} \times 100 \mathrm{~cm} \times 40 \mathrm{~cm}$ ) divided into a series of squares of black and white color. The number of squares moved by each animal was counted for 5 min at $0,30,60,90$ and 120 min during the study period. Number of movement reduced by extracts revealed the extant of depressant, and the percentage inhibition of square movement was calculated at $120 \mathrm{~min}$ by the following formula:

$\%$ Inhibition $=\left[\left(\mathrm{N}_{0}-\mathrm{N}_{\mathrm{S}}\right) / \mathrm{N}_{0}\right] \times 100$

Where, $\mathrm{N}_{0}$ is the average number of square traveled by control group, $\mathrm{N}_{S}$ is the average number of square traveled by treatment or standard group.

\subsubsection{Hole cross test}

The method described by Takagi et al. [24, 25], was applied for this study. According to this method, eighty four albino mice were divided into fourteen groups $(n=6)$. As like open field test, vehicle (1\% tween 80 in water, p.o.), diazepam (2 mg/kg, p.o.), AEDFL (50, 100 and $200 \mathrm{mg} / \mathrm{kg}$, p.o.), EEDFL (50, 100 and 200 $\mathrm{mg} / \mathrm{kg}$, p.o.), CEDFL (50, 100 and $200 \mathrm{mg} / \mathrm{kg}$, p.o.) and PEDFL (50, 100 and $200 \mathrm{mg} / \mathrm{kg}$, p.o.) were administered to the respective groups. Then gradually all animals were placed in a chamber of a box having a size of $30 \times 20 \times 14 \mathrm{~cm}$ where a partition was made at the middle of the cage. A hole of $3 \mathrm{~cm}$ diameter was made at a height of 7.5 $\mathrm{cm}$ in the center of the cage. After the treatment, the total number of passages of each mouse through the hole from one chamber to other was counted for a period of $5 \mathrm{~min}$ on $0,30,60,90$ and 120 min during the study period. CNS depressant activity was assessed by the reduced number of passages of mice through the hole and percentage protection of movement was calculated at 120 min by the following formula:

$\%$ Inhibition $=\left[\left(\mathrm{N}_{0}-\mathrm{N}_{\mathrm{S}}\right) / \mathrm{N}_{0}\right] \times 100$

Where, $\mathrm{N}_{0}$ is the average number of passage by control group, $\mathrm{N}_{\mathrm{S}}$ is the average number of passage by treatment or standard group.

\subsection{Statistical analysis}

All the values were expressed as the mean \pm SEM (Standard Error Mean) of three replicate experiments $(n=6$ mice per group). The analyses were performed by using SPSS statistical package for WINDOWS (version 15.0; SPSS Inc, Chicago) and $\mathrm{P}^{\mathrm{b}}<0.01, \quad \mathrm{P}^{\mathrm{a}}<0.001 \quad$ were considered to be statistically significant compared to vehicle control group. Statistical significance (p) calculated by ANOVA followed by Dunnett 's test.

\section{Results}

\subsection{Analgesic activity}

\subsubsection{Acetic acid induced writhing method}

The result in Table 1 showed the effect of oral administration of AEDFL, EEDFL, CEDFL and PEDFL $(50,100,200 \mathrm{mg} / \mathrm{kg})$ in acetic acid-induced abdominal writhing test. All the extracts have reduced writhing-response significantly $\left(\mathrm{P}^{\mathrm{a}}<0.001\right)$ in dose dependant fashion. Morphine sulfate $(5 \mathrm{mg} / \mathrm{kg})$ was used as standard drug that reduced writhing number to $5.39 \pm 1.52$ and exhibited maximum inhibition $(88.47 \%)$. PEDFL showed maximum analgesic effect among the extracts. It exhibited $44.98 \%$ (25.72 \pm 3.20$), 64.86 \%(16.42 \pm 2.20)$ and $77.45 \%$ $(10.54 \pm 1.43)$ inhibition of abdominal writhing at 
50,100 and $200 \mathrm{mg} / \mathrm{kg}$, p.o. doses. AEDFL, EEDFL and CEDFL showed $57.75 \%$ $(19.75 \pm 4.21), 66.73 \%(15.55 \pm 2.10)$ and $61.06 \%$ $(18.20 \pm 2.10)$ at $200 \mathrm{mg} / \mathrm{kg}$ dose respectively. The order of analgesic potential among the four extracts was PEDFL> EEDFL> CEDFL> AEDFL.

Table 1: Analgesic effect of D. falcata leaves extracts and morphine sulfate in acetic acid-induced writhing test.

\begin{tabular}{cccc} 
Sample & $\begin{array}{c}\text { Dose } \\
\text { mg/kg,p.o. }\end{array}$ & Writhing number & $\begin{array}{c}\text { Percentage inhibition of } \\
\text { writhing }\end{array}$ \\
\hline Control (vehicle) & $0.1 \mathrm{ml} / \mathrm{mice}$ & $46.75 \pm 4.50$ & 00 \\
Morphine sulfate & $10 \mathrm{mg} / \mathrm{kg}$ & $5.39 \pm 1.52^{\mathrm{a}}$ & 88.47 \\
AEDFL & 50 & $37.25 \pm 4.18^{\mathrm{a}}$ & 20.32 \\
& 100 & $27.60 \pm 3.40^{\mathrm{a}}$ & 40.96 \\
& 200 & $19.75 \pm 4.21^{\mathrm{a}}$ & 57.75 \\
EEDFL & 50 & $30.32 \pm 3.45^{\mathrm{a}}$ & 35.14 \\
& 100 & $21.30 \pm 3.23^{\mathrm{a}}$ & 54.43 \\
& 200 & $15.55 \pm 2.10^{\mathrm{a}}$ & 66.73 \\
CEDFL & 50 & $34.54 \pm 3.45^{\mathrm{a}}$ & 26.11 \\
& 100 & $24.12 \pm 4.50^{\mathrm{a}}$ & 48.40 \\
& 200 & $18.20 \pm 2.10^{\mathrm{a}}$ & 61.06 \\
PEDFL & 50 & $25.72 \pm 3.20^{\mathrm{a}}$ & 44.98 \\
& 100 & $16.42 \pm 2.20^{\mathrm{a}}$ & 64.86 \\
& 200 & $10.54 \pm 1.43^{\mathrm{a}}$ & 77.45
\end{tabular}

Each value is presented as mean \pm SEM $(n=6) ; p^{a}<0.001$ compared with the control group. ANOVA followed by Dunnett's test is done in SPSS version 15.0

\subsubsection{Tail immersion method}

In the radiant heat tail-immersion test, the leaves extracts prolonged the heat stress tolerance capacity of treated mice. The tail-withdrawal reflex time of mice by the hot water-induced pain was significant after administration of AEDFL, EEDFL, CEDFL and PEDFL. All the extracts at 50,100 and $200 \mathrm{mg} / \mathrm{kg}$ doses showed statistically significant $\left(\mathrm{p}^{\mathrm{b}}<0.01, \mathrm{p}^{\mathrm{a}}<0.001\right)$ elongation of reaction time with the increase order of dose compared with control group (vehicle). Morphine sulfate $(5 \mathrm{mg} / \mathrm{kg}$,p.o.) was used as

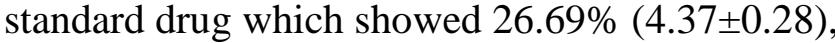
$45.05 \%(5.78 \pm 0.10), 51.17 \%(6.25 \pm 0.30)$ and
94.53\% (9.58 \pm 0.45$) \mathrm{MPE}$ at $30 \mathrm{~min}, 60 \mathrm{~min}, 90$ min and 120 min respectively. PEDFL showed highest and AEDFL showed lowest effects among the extracts. At $200 \mathrm{mg} / \mathrm{kg}$ dose, PEDFL exhibited $24.20 \% \quad(4.02 \pm 0.97), \quad 49.04 \%$ (5.98 \pm 0.66$), 60.45 \%(6.88 \pm 0.61)$ and $82.12 \%$ (8.59 \pm 0.24$)$ MPE; EEDFL exhibited $17.77 \%$ $(4.08 \pm 0.47), \quad 38.81 \% \quad(5.59 \pm 0.91), \quad 52.36 \%$ $(6.57 \pm 0.65)$ and $75.00 \%$ MPE (8.20 \pm 0.6$)$; CEDFL exhibited $25.40 \%$ (4.07 \pm 0.19$), 30.50 \%$ $(4.47 \pm 0.73), \quad 49.87 \% \quad(6.52 \pm 0.52), \quad 57.23 \%$ (6.6 \pm 0.08$) \mathrm{MPE} ; \quad$ AEDFL showed $13.92 \%$ $(3.26 \pm 0.62), \quad 27.45 \% \quad(4.32 \pm 0.4), \quad 45.40 \%$ $(5.725 \pm 0.4)$ and $51.53 \%$ MPE $(6.205 \pm 0.41)$ at 30 
min, $60 \mathrm{~min}, 90 \mathrm{~min}$ and 120 respectively. The EEDFL $>$ CEDFL > AEDFL (Table 2).

order of tail withdrawal reflex time was PEDFL>

Table 2: Analgesic effect of $D$. falcata leaves extracts and morphine sulfate in tail immersion test.

\begin{tabular}{|c|c|c|c|c|c|c|}
\hline \multirow[b]{2}{*}{ Sample } & \multirow[b]{2}{*}{$\begin{array}{c}\text { Dose } \\
\text { mg/kg.p.o. }\end{array}$} & \multicolumn{5}{|c|}{ Response time (s) (\%MPE) } \\
\hline & & 0 min & $30 \mathrm{~min}$ & $60 \min$ & $90 \mathrm{~min}$ & $120 \mathrm{~min}$ \\
\hline Vehicle & $0.1 \mathrm{ml} /$ mice & $2.13 \pm 0.19$ & $2.10 \pm 0.05$ & $2.24 \pm 0.29$ & $2.5 \pm 0.40$ & $2.80 \pm 0.09$ \\
\hline \multirow[t]{2}{*}{$\begin{array}{l}\text { Morphine } \\
\text { sulfate }\end{array}$} & 5 & $2.32 \pm 0.35$ & $\begin{array}{c}4.37 \pm 0.28^{\mathrm{a}} \\
\quad(26.69)\end{array}$ & $\begin{array}{c}5.78 \pm 0.10^{\mathrm{a}} \\
(45.05)\end{array}$ & $\begin{array}{c}6.25 \pm 0.30^{\mathrm{a}} \\
\quad(51.17)\end{array}$ & $\begin{array}{c}9.58 \pm 0.45^{\mathrm{a}} \\
(94.53)\end{array}$ \\
\hline & 50 & $2.25 \pm 0.23$ & $\begin{array}{c}2.37 \pm 0.50^{\mathrm{b}} \\
\quad(1.54)\end{array}$ & $\begin{array}{c}3.20 \pm 0.3^{\mathrm{a}} \\
(12.25)\end{array}$ & $\begin{array}{c}4.19 \pm 0.24^{\mathrm{a}} \\
(25.03)\end{array}$ & $\begin{array}{c}4.49 \pm 0.33^{\mathrm{a}} \\
(28.90)\end{array}$ \\
\hline \multirow[t]{3}{*}{ AEDFL } & 100 & $2.29 \pm 0.2$ & $\begin{array}{c}3.07 \pm 0.52^{\mathrm{a}} \\
\quad(10.11)\end{array}$ & $\begin{array}{c}3.42 \pm 0.58^{\mathrm{a}} \\
\quad(14.65)\end{array}$ & $\begin{array}{c}4.8 \pm 0.2^{\mathrm{a}} \\
(32.55)\end{array}$ & $\begin{array}{c}5.47 \pm 0.4^{\mathrm{a}} \\
(41.24)\end{array}$ \\
\hline & 200 & $2.17 \pm 0.42$ & $\begin{array}{l}3.26 \pm 0.62^{\mathrm{a}} \\
(13.92)\end{array}$ & $\begin{array}{l}4.32 \pm 0.4^{\mathrm{a}} \\
\quad(27.45\end{array}$ & $\begin{array}{l}5.725 \pm 0.4^{\mathrm{a}} \\
(45.40)\end{array}$ & $\begin{array}{c}6.205 \pm 0.41^{\mathrm{a}} \\
(51.53)\end{array}$ \\
\hline & 50 & $2.27 \pm 0.1$ & $\begin{array}{c}2.85 \pm 0.05^{\mathrm{b}} \\
(7.50)\end{array}$ & $\begin{array}{c}3.8 \pm 0.3^{\mathrm{a}} \\
(19.79)\end{array}$ & $\begin{array}{l}4.41 \pm 0.50^{\mathrm{a}} \\
\quad(27.68)\end{array}$ & $\begin{array}{c}5.22 \pm 0.25^{\mathrm{a}} \\
38.18)\end{array}$ \\
\hline \multirow[t]{3}{*}{ EEDFL } & 100 & $2.37 \pm 0.2$ & $\begin{array}{c}3.42 \pm 0.42^{\mathrm{a}} \\
\quad(13.76)\end{array}$ & $\begin{array}{c}4.24 \pm 0.2^{\mathrm{a}} \\
(24.50)\end{array}$ & $\begin{array}{c}5.19 \pm 0.22^{\mathrm{a}} \\
(36.95)\end{array}$ & $\begin{array}{c}6.17 \pm 0.11^{\mathrm{a}} \\
(49.80)\end{array}$ \\
\hline & 200 & $2.8 \pm 0.36$ & $\begin{array}{c}4.08 \pm 0.47^{\mathrm{a}} \\
(17.77)\end{array}$ & $\begin{array}{c}5.59 \pm 0.91^{\mathrm{a}} \\
(38.81)\end{array}$ & $\begin{array}{c}6.57 \pm 0.65^{\mathrm{a}} \\
(52.36)\end{array}$ & $\begin{array}{c}8.20 \pm 0.6^{\mathrm{a}} \\
(75.00)\end{array}$ \\
\hline & 50 & $2.25 \pm 0.1$ & $\begin{array}{l}2.85 \pm 0.05^{\mathrm{b}} \\
(7.74)\end{array}$ & $\begin{array}{c}3.05 \pm 0.42^{\mathrm{a}} \\
\quad(10.32)\end{array}$ & $\begin{array}{c}4.17 \pm 0.2^{\mathrm{a}} \\
(24.77)\end{array}$ & $\begin{array}{c}4.62 \pm 0.3^{\mathrm{a}} \\
(30.58)\end{array}$ \\
\hline \multirow[t]{3}{*}{ CEDFL } & 100 & $2.34 \pm 0.45$ & $\begin{array}{c}3.14 \pm 0.34^{\mathrm{a}} \\
(10.44)\end{array}$ & $\begin{array}{c}3.99 \pm 0.3^{\mathrm{a}} \\
(21.54)\end{array}$ & $\begin{array}{c}5.00 \pm 0.42^{\mathrm{a}} \\
(34.72)\end{array}$ & $\begin{array}{c}5.79 \pm 0.54^{\mathrm{a}} \\
(45.03)\end{array}$ \\
\hline & 200 & $2.05 \pm 0.42$ & $\begin{array}{c}4.07 \pm 0.19^{\mathrm{a}} \\
(25.40)\end{array}$ & $\begin{array}{c}4.475 \pm 0.73^{\mathrm{a}} \\
(30.50)\end{array}$ & $\begin{array}{c}6.525 \pm 0.52^{\mathrm{a}} \\
\quad(49.87)\end{array}$ & $\begin{array}{c}6.6 \pm 0.08^{\mathrm{a}} \\
(57.23)\end{array}$ \\
\hline & 50 & $2.03 \pm 0.35$ & $\begin{array}{c}2.74 \pm 0.27^{b} \\
\quad(8.90)\end{array}$ & $\begin{array}{c}3.49 \pm 0.02^{\mathrm{a}} \\
(18.31)\end{array}$ & $\begin{array}{c}4.19 \pm 0.50^{\mathrm{a}} \\
(27.10)\end{array}$ & $\begin{array}{c}5.30 \pm 0.56^{\mathrm{a}} \\
(41.02)\end{array}$ \\
\hline \multirow[t]{2}{*}{ PEDFL } & 100 & $2.07 \pm 0.35$ & $\begin{array}{c}3.8 \pm 0.25^{\mathrm{a}} \\
21.81)\end{array}$ & $\begin{array}{c}4.47 \pm 0.03^{\mathrm{a}} \\
\quad(30.26)\end{array}$ & $\begin{array}{c}5.68 \pm 0.1^{\mathrm{a}} \\
(45.52)\end{array}$ & $\begin{array}{c}6.59 \pm 0.52^{\mathrm{a}} \\
(56.99)\end{array}$ \\
\hline & 200 & $2.11 \pm 0.61$ & $\begin{array}{c}4.02 \pm 0.97^{\mathrm{a}} \\
(24.20)\end{array}$ & $\begin{array}{c}5.98 \pm 0.66^{\mathrm{a}} \\
(49.04)\end{array}$ & $\begin{array}{c}6.88 \pm 0.61^{\mathrm{a}} \\
(60.45)\end{array}$ & $\begin{array}{c}8.59 \pm 0.24^{\mathrm{a}} \\
(82.12)\end{array}$ \\
\hline
\end{tabular}

Each value is presented as mean \pm SEM $(n=6) ; p^{b}<0.01, p^{a}<0.001$ compared with the vehicle control group. ANOVA followed by Dunnett's test is done in SPSS version 15.0

\subsection{Anti-inflammatory activity}

Table 3 represents the anti- inflammatory activity of AEDFL, EEDFL, CEDFL and PEDFL. All the extracts showed statistically significant $\left(\mathrm{p}^{\mathrm{b}}<0.01, \mathrm{p}^{\mathrm{a}}<0.001\right)$ antiinflammatory activity as dose dependant manner. Data are presented as volume (mL) of paw edema and inhibition (\%) of it (paw edema volume). Ibuprofen $(5 \mathrm{mg} / \mathrm{kg})$ was used as standard drug which showed maximum inhibition $(96.34 \%)$ of paw volume at 4 hour of observation. PEDFL exhibited $\quad 18.98 \% \quad(0.64 \pm 0.01), \quad 25.31 \%$ $(0.59 \pm 0.01)$ and $36.70 \%(0.5 \pm 0.03)$ inhibition at $1 \mathrm{~h}$ and it (inhibition) continued up to the fifth observation $(4 \mathrm{~h})$ where this fraction produced $70.73 \% \quad(0.24 \pm 0.03), 81.70 \% \quad(0.15 \pm 0.01)$ and $90.24 \%(0.08 \pm 0.02)$ inhibition of paw edema volume at $50,100,200 \mathrm{mg} / \mathrm{kg}$ dose respectively. 
This fraction exhibited highest anti-inflammatory potential among the four extracts. After oral administration of $200 \mathrm{mg} / \mathrm{kg}$ dose EEDFL showed $27.84 \%, 56.25 \%, 76.54 \%$ and $87.80 \%$ inhibition; AEDFL exhibited $26.58 \%, 48.75 \%, 71.60 \%$ and
80.48\% inhibition, as well as CEDFL showed $25.31 \%, 46.25 \%, 69.13 \%$ and $76.82 \%$ inhibition of paw volume at $1 \mathrm{~h}, 2 \mathrm{~h}, 3 \mathrm{~h}$ and $4 \mathrm{~h}$ respectively. The order of paw volume inhibition was PEDFL $>$ EEDFL $>$ AEDFL $>$ CEDFL.

Table 3: Effect of p.o. administration of $D$. falcata leaves extracts on carrageenan-induced paw edema.

\begin{tabular}{lcccccc}
\hline \multirow{2}{*}{ Sample } & & \multicolumn{5}{c}{ Mean edema volume in mL (Percentage inhibition) } \\
\cline { 2 - 7 } & $\begin{array}{c}\text { Dose } \\
\mathrm{mg} / \mathrm{kg}\end{array}$ & $0 \mathrm{~h}$ & $1 \mathrm{~h}$ & $2 \mathrm{~h}$ & $3 \mathrm{~h}$ & $4 \mathrm{~h}$ \\
\hline Control & $0.1 \mathrm{ml}$ & $0.77 \pm 0.03$ & $0.79 \pm 0.02$ & $0.80 \pm 0.02$ & $0.81 \pm 0.01$ & $0.82 \pm 0.03$ \\
Ibuprofen & 10 & $0.79 \pm 0.01$ & $0.46 \pm 0.01^{\mathrm{a}}(41.72)$ & $0.26 \pm 0.02^{\mathrm{a}}(67.50)$ & $0.05 \pm 0.03^{\mathrm{a}}(93.82)$ & $0.03 \pm 0.01^{\mathrm{a}}(96.34)$ \\
& 50 & $0.79 \pm 0.02$ & $0.69 \pm 0.01^{\mathrm{b}}(12.65)$ & $0.61 \pm 0.01^{\mathrm{b}}(23.75)$ & $0.49 \pm 0.01^{\mathrm{a}}(39.50)$ & $0.3 \pm 0.01^{\mathrm{a}}(63.41)$ \\
AEDFL & 100 & $0.78 \pm 0.01$ & $0.64 \pm 0.01^{\mathrm{b}}(18.98)$ & $0.49 \pm 0.02^{\mathrm{a}}(38.75)$ & $0.32 \pm 0.03^{\mathrm{a}}(60.49)$ & $0.29 \pm 0.01^{\mathrm{a}}(64.63)$ \\
& 200 & $0.8 \pm 0.01$ & $0.58 \pm 0.02^{\mathrm{a}}(26.58)$ & $0.41 \pm 0.03^{\mathrm{a}}(48.75)$ & $0.23 \pm 0.01^{\mathrm{a}}(71.60)$ & $0.16 \pm 0.01^{\mathrm{a}}(80.48)$ \\
& 50 & $0.81 \pm 0.03$ & $0.65 \pm 0.02^{\mathrm{b}}(15.18)$ & $0.58 \pm 0.01^{\mathrm{a}}(27.50)$ & $0.39 \pm 0.01^{\mathrm{a}}(51.85)$ & $0.27 \pm 0.03^{\mathrm{a}}(67.07)$ \\
EEDFL & 100 & $0.79 \pm 0.03$ & $0.6 \pm 0.01^{\mathrm{b}}(24.05)$ & $0.47 \pm 0.02^{\mathrm{a}}(41.25)$ & $0.29 \pm 0.01^{\mathrm{a}}(64.19)$ & $0.18 \pm 0.01^{\mathrm{a}}(78.04)$ \\
& 200 & $0.75 \pm 0.01$ & $0.57 \pm 0.02^{\mathrm{a}}(27.84)$ & $0.35 \pm 0.01^{\mathrm{a}}(56.25)$ & $0.19 \pm 0.02^{\mathrm{a}}(76.54)$ & $0.10 \pm 0.02^{\mathrm{a}}(87.80)$ \\
& 50 & $0.82 \pm 0.02$ & $0.73 \pm 0.03(7.59)$ & $0.63 \pm 0.01^{\mathrm{b}}(21.25)$ & $0.51 \pm 0.01^{\mathrm{a}}(37.03)$ & $0.35 \pm 0.01^{\mathrm{a}}(57.31)$ \\
CEDFL & 100 & $0.8 \pm 0.01$ & $0.67 \pm 0.02^{\mathrm{b}}(15.18)$ & $0.53 \pm 0.01^{\mathrm{a}}(33.75)$ & $0.37 \pm 0.03^{\mathrm{a}}(54.32)$ & $0.32 \pm 0.02^{\mathrm{a}}(60.97)$ \\
& 200 & $0.78 \pm 0.02$ & $0.59 \pm 0.02^{\mathrm{a}}(25.31)$ & $0.43 \pm 0.03^{\mathrm{a}}(46.25)$ & $0.25 \pm 0.01^{\mathrm{a}}(69.13)$ & $0.19 \pm 0.02^{\mathrm{a}}(76.82)$ \\
& 50 & $0.8 \pm 0.01$ & $0.64 \pm 0.01(18.98)$ & $0.5 \pm 0.01^{\mathrm{a}}(37.5)$ & $0.36 \pm 0.01^{\mathrm{a}}(55.55)$ & $0.24 \pm 0.03^{\mathrm{a}}(70.73)$ \\
PEDFL & 100 & $0.76 \pm 0.03$ & $0.59 \pm 0.01^{\mathrm{a}}(25.31)$ & $0.43 \pm 0.02^{\mathrm{a}}(46.25)$ & $0.27 \pm 0.03^{\mathrm{a}}(66.66)$ & $0.15 \pm 0.01^{\mathrm{a}}(81.70)$ \\
& & & & & & \\
& 200 & $0.82 \pm 0.02$ & $0.5 \pm 0.03^{\mathrm{a}}(36.70)$ & $0.3 \pm 0.03^{\mathrm{a}}(62.50)$ & $0.15 \pm 0.01^{\mathrm{a}}(81.48)$ & $0.08 \pm 0.02^{\mathrm{a}}(90.24)$
\end{tabular}

Each value is presented as mean \pm SEM $(n=6) ; p^{b}<0.01, p^{a}<0.001$ compared with the vehicle control group. ANOVA followed by Dunnett's test is done in SPSS version 15.0

\subsection{CNS depression activity}

\subsubsection{Open field test}

Aqueous, ethanol, chloroform and petroleum ether extracts (AEDFL, EEDFL, CEDFL and PEDFL) of the D. falcata leaves exhibited statistically significant $\left(\mathrm{p}^{\mathrm{a}}<0.001\right)$ reduction of movements (square of open field) of the test mice for respective group compared with vehicle control group. The results were significant from second observation (30 $\mathrm{min})$ and continued up to fifth observation $(120 \mathrm{~min})$ in dose dependant manner $(200 \mathrm{mg}>100>50 \mathrm{mg})$. Among the four extracts, EEDFL (200mg) showed maximum effect which is similar with the effect of standard drug diazepam (2mg). EEDFL (200mg) showed $174.67 \pm 8.70$ movements at 0 min of treatment. 30 min after oral administration, sharp reduction $\left(\mathrm{p}^{\mathrm{a}}<0.001\right)$ of locomotion $(43.14 \pm 6.26)$ was observed by the extract, and in fifth observation (120min) movement number was only 4.09 \pm 3.61 where inhibition of movement was $97.33 \%$. On 
the other hand, AEDFL (200mg) showed least effect. It showed 175.35 $\pm 9.33,110.23 \pm 6.3$ and $16.42 \pm 4.33^{\mathrm{a}}$ movements in $0 \mathrm{~min}, 30 \mathrm{~min}$ and 120 min respectively, and showed $89.30 \%$ inhibition after $120 \mathrm{~min}$. Standard drug diazepam $(2 \mathrm{mg}$ ) exhibited $178.21 \pm 7.32$ movement at $0 \mathrm{~min}$.
It showed $67.29 \pm 8.42$ and $4.39 \pm 0.55$ movements in $30 \mathrm{~min}$ and $120 \mathrm{~min}$ respectively where inhibition of movement was $97.14 \%$ (120min). The order of CNS depressant effect among the four extracts was EEDFL> PEDFL>CEDFL> AEDFL (Table 4).

Table 4: CNS depressant activity of different fractions of $D$. falcata leaves by open field test in mice.

\begin{tabular}{|c|c|c|c|c|c|c|c|}
\hline \multirow{2}{*}{ Sample } & \multirow[b]{2}{*}{$\begin{array}{l}\text { Dose } \\
\mathrm{mg} / \mathrm{kg} \\
\text {.p.o. }\end{array}$} & \multicolumn{5}{|c|}{ Number of movements } & \multirow{2}{*}{$\begin{array}{l}\% \text { Inhibition } \\
\text { of } \\
\text { movements } \\
\text { after } 120 \\
\text { min }\end{array}$} \\
\hline & & $0 \min$ & $30 \mathrm{~min}$ & $60 \mathrm{~min}$ & $90 \min$ & $120 \mathrm{~min}$ & \\
\hline $\begin{array}{l}\text { Control } \\
\text { (vehicle) }\end{array}$ & $0.1 \mathrm{ml}$ & $\begin{array}{c}180.45 \pm 7.6 \\
3\end{array}$ & $176.67 \pm 6.11$ & $173.82 \pm 6.83$ & $167.72 \pm 5.58$ & $153.58 \pm 5.37$ & -- \\
\hline \multirow[t]{2}{*}{ Diazepam } & 2 & $\begin{array}{c}178.21 \pm 7.3 \\
2\end{array}$ & $67.29 \pm 8.42^{\mathrm{a}}$ & $23.45 \pm 3.02^{\mathrm{a}}$ & $8.23 \pm 2.12^{\mathrm{a}}$ & $4.39 \pm 0.55^{\mathrm{a}}$ & 97.14 \\
\hline & 50 & $\begin{array}{c}173.63 \pm 9.1 \\
2\end{array}$ & $\begin{array}{c}137.68 \pm 6.30 \\
\text { a }\end{array}$ & $57.22 \pm 8.07^{\mathrm{a}}$ & $49.56 \pm 6.23^{\mathrm{a}}$ & $37.22 \pm 4.34^{\mathrm{a}}$ & 75.75 \\
\hline \multirow[t]{3}{*}{ AEDFL } & 100 & $\begin{array}{c}169.45 \pm 8.4 \\
0\end{array}$ & $125.20 \pm 8.2^{\mathrm{a}}$ & $43.32 \pm 8.42^{\mathrm{a}}$ & $32.37 \pm 7.47^{\mathrm{a}}$ & $21.30 \pm 5.14^{\mathrm{a}}$ & 86.13 \\
\hline & 200 & $\begin{array}{c}175.35 \pm 9.3 \\
3\end{array}$ & $110.23 \pm 6.3^{\mathrm{a}}$ & $38.12 \pm 7.02^{\mathrm{a}}$ & $18.04 \pm 5.43^{\mathrm{a}}$ & $16.42 \pm 4.33^{\mathrm{a}}$ & 89.30 \\
\hline & 50 & $\begin{array}{c}177.20 \pm 9.5 \\
0\end{array}$ & $77.35 \pm 8.30^{\mathrm{a}}$ & $40.27 \pm 6.35^{\mathrm{a}}$ & $27.56 \pm 5.30^{\mathrm{a}}$ & $17.65 \pm 3.10^{\mathrm{a}}$ & 88.50 \\
\hline \multirow[t]{3}{*}{ EEDFL } & 100 & $\begin{array}{c}170.27 \pm 8.7 \\
7\end{array}$ & $58.87 \pm 6.33^{\mathrm{a}}$ & $32.28 \pm 5.55^{\mathrm{a}}$ & $16.75 \pm 6.32^{\mathrm{a}}$ & $9.25 \pm 2.12^{\mathrm{a}}$ & 93.97 \\
\hline & 200 & $\begin{array}{c}174.67 \pm 8.7 \\
0\end{array}$ & $43.14 \pm 6.26^{\mathrm{a}}$ & $21.49 \pm 4.50^{\mathrm{a}}$ & $12.29 \pm 3.34^{\mathrm{a}}$ & $4.09 \pm 3.61^{\mathrm{a}}$ & 97.33 \\
\hline & 50 & $\begin{array}{c}179.31 \pm 9.8 \\
0\end{array}$ & $88.41 \pm 9.93^{\mathrm{a}}$ & $59.50 \pm 7.57^{\mathrm{a}}$ & $37.45 \pm 6.43^{\mathrm{a}}$ & $22.10 \pm 3.13^{\mathrm{a}}$ & 85.61 \\
\hline \multirow[t]{3}{*}{ CEDFL } & 100 & $\begin{array}{c}173.29 \pm 7.5 \\
0\end{array}$ & $71.30 \pm 7.83^{\mathrm{a}}$ & $47.54 \pm 6.50^{\mathrm{a}}$ & $26.43 \pm 5.01^{\mathrm{a}}$ & $13.39 \pm 2.56^{\mathrm{a}}$ & 91.28 \\
\hline & 200 & $\begin{array}{c}182.34 \pm 7.2 \\
3\end{array}$ & $49.20 \pm 6.21^{\mathrm{a}}$ & $35.5 \pm 3.87^{\mathrm{a}}$ & $22.43 \pm 4.01^{\mathrm{a}}$ & $10.23 \pm 2.33^{\mathrm{a}}$ & 93.33 \\
\hline & 50 & $\begin{array}{c}183.88 \pm 9.6 \\
0\end{array}$ & $79.27 \pm 8.23^{\mathrm{a}}$ & $50.20 \pm 7.56^{\mathrm{a}}$ & $41.08 \pm 6.78^{\mathrm{a}}$ & $23.07 \pm 3.10^{\mathrm{a}}$ & 84.97 \\
\hline \multirow[t]{2}{*}{ PEDFL } & 100 & $\begin{array}{c}183.45 \pm 5.5 \\
4\end{array}$ & $73.38 \pm 5.22^{\mathrm{a}}$ & $39.38 \pm 3.54^{\mathrm{a}}$ & $25.84 \pm 3.20^{\mathrm{a}}$ & $11.36 \pm 2.02^{\mathrm{a}}$ & 92.60 \\
\hline & 200 & $\begin{array}{c}170.59 \pm 5.2 \\
9\end{array}$ & $45.30 \pm 5.69^{\mathrm{a}}$ & $27.40 \pm 4.06^{\mathrm{a}}$ & $17.11 \pm 3.13^{\mathrm{a}}$ & $6.79 \pm 2.38^{\mathrm{a}}$ & 95.57 \\
\hline
\end{tabular}

Each value is presented as the mean \pm SEM $(n=6) ; p^{a}<0.001$ compared with the vehicle control group. ANOVA followed by Dunnett's test is done in SPSS version 15.0

\subsubsection{Hole-cross test}

In the hole-cross test, the number of passes of tested mice through the hole was reduced significantly $\left(\mathrm{p}^{\mathrm{b}}<0.01, \mathrm{p}^{\mathrm{a}}<0.001\right)$ by AEDFL, EEDFL, CEDFL and PEDFL compared with control group. Except 50mg of AEDFL, all of 
the fractions and crude ethanol extracts exhibited CNS depressant potential from second observation $(30 \mathrm{~min})$ to fifth observation (120 $\mathrm{min})$ in dose dependant fashion (200mg $>100>50 \mathrm{mg})$. Percentage inhibition of movements was calculated after $120 \mathrm{~min}$ of drug and extracts treatment in respect to control (vehicle) group. In case of diazepam treated mice, number of movements was $15.20 \pm 1.55$ in 0 min which sharply reduced to $4.28 \pm 1.12$ in $30 \mathrm{~min}$. This reduction of movements was continued up to $120 \mathrm{~min}$ where inhibition of movements was
91.94\%. Among the four extracts, crude ethanol portion showed maximum inhibition $(85.91 \%)$ whereas aqueous extract exhibited minimum inhibition $(71.40 \%)$ at $200 \mathrm{mg} / \mathrm{kg}$ dose. After 30 min of oral administration of AEDFL, EEDFL, CEDFL and PEDFL, number of movements were $10.13 \pm 2.15,5.28 \pm 1.92,7.10 \pm 0.30$ and $6.20 \pm 1.0$ which reached to $4.08 \pm 1.55,2.01 \pm 0.22$, $3.39 \pm 0.53$ and $2.59 \pm 1.54$ respectively after 120 $\mathrm{min}$ at $200 \mathrm{mg}$ dose. The order of CNS depressant activity was EEDFL> PEDFL> CEDFL> AEDFL (Table 5).

Table 5: CNS depressant activity of different fractions of $D$. falcata leaves by hole cross test in mice.

\section{Number of movements}

Sample Dose

$\mathrm{mg} / \mathrm{kg}$ p.o.

\begin{tabular}{lccccccc}
\hline Control & $0.1 \mathrm{ml}$ & $16.37 \pm 1.44$ & $15.56 \pm 1.50$ & $14.71 \pm 2.32$ & $14.10 \pm 2.62$ & $14.27 \pm 1.10$ & -- \\
Diazepam & 2 & $15.20 \pm 1.55$ & $4.28 \pm 1.12^{\mathrm{a}}$ & $2.34 \pm 0.58^{\mathrm{a}}$ & $1.95 \pm 0.22^{\mathrm{a}}$ & $1.15 \pm 0.01^{\mathrm{a}}$ & 91.94 \\
& 50 & $17.35 \pm 3.42$ & $15.30 \pm 3.15$ & $11.15 \pm 2.50^{\mathrm{a}}$ & $9.10 \pm 1.15^{\mathrm{a}}$ & $8.20 \pm 2.12^{\mathrm{a}}$ & 42.53 \\
AEDFL & 100 & $19.45 \pm 1.50$ & $13.33 \pm 2.24^{\mathrm{b}}$ & $9.45 \pm 1.50^{\mathrm{a}}$ & $6.5 \pm 1.02^{\mathrm{a}}$ & $5.89 \pm 1.76^{\mathrm{a}}$ & 58.72 \\
& 200 & $18.5 \pm 1.55$ & $11.45 \pm 2.10^{\mathrm{a}}$ & $6.5 \pm 0.50^{\mathrm{a}}$ & $5.1 \pm 0.43^{\mathrm{a}}$ & $4.08 \pm 1.55^{\mathrm{a}}$ & 71.40 \\
& 50 & $15.5 \pm 1.11$ & $10.13 \pm 2.15^{\mathrm{a}}$ & $6.17 \pm 1.10^{\mathrm{a}}$ & $5.75 \pm 1.25^{\mathrm{a}}$ & $4.57 \pm 0.55^{\mathrm{a}}$ & 67.97 \\
EEDFL & 100 & $16.5 \pm 1.11$ & $7.39 \pm 1.45^{\mathrm{a}}$ & $5.47 \pm 0.80^{\mathrm{a}}$ & $5.15 \pm 1.02^{\mathrm{a}}$ & $3.39 \pm 0.32^{\mathrm{a}}$ & 76.24 \\
& 200 & $14.25 \pm 1.50$ & $5.28 \pm 1.92^{\mathrm{a}}$ & $3.39 \pm 0.50^{\mathrm{a}}$ & $3.10 \pm 0.10^{\mathrm{a}}$ & $2.01 \pm 0.22^{\mathrm{a}}$ & 85.91 \\
& 50 & $15.5 \pm 2.32$ & $12.12 \pm 2.51^{\mathrm{a}}$ & $10.2 \pm 1.64^{\mathrm{a}}$ & $9.54 \pm 2.10^{\mathrm{a}}$ & $7.25 \pm 1.23^{\mathrm{a}}$ & 49.19 \\
CEDFL & 100 & $14.5 \pm 1.32$ & $9.0 \pm 1.55^{\mathrm{a}}$ & $7.0 \pm 0.52^{\mathrm{a}}$ & $6.81 \pm 1.01^{\mathrm{a}}$ & $5.39 \pm 1.52^{\mathrm{a}}$ & 62.22 \\
& 200 & $15.68 \pm 1.40$ & $7.10 \pm 0.30^{\mathrm{a}}$ & $5.5 \pm 0.02^{\mathrm{a}}$ & $3.76 \pm 0.01^{\mathrm{a}}$ & $3.39 \pm 0.53^{\mathrm{a}}$ & 76.24
\end{tabular}

Each value is presented as mean \pm SEM $(n=6) ; p^{b}<0.01, p^{a}<0.001$ compared with the vehicle control group. ANOVA followed by Dunnett's test is done in SPSS version 15.0 


\section{Discussion}

Results of the present study showed that the D. falcata leaves extracts have marked analgesic, anti-inflammatory and CNS depressant activities that have disclosed the effectiveness of the plant in folk medicine. Pain and inflammation are associated with the pathophysiology of various clinical conditions such as arthritis, cancer, vascular diseases, asthma, multiple sclerosis, colitis, inflammatory bowel disease and atherosclerosis. Many natural products are used in traditional medical systems to relieve the symptoms from pain and inflammation [26].

Acetic acid-induced writhing and heatinduced tail immersion tests are simple, reliable and well recommended protocols in evaluating medicinal agents for their analgesic property. Analgesics can act both on peripheral or central nervous system. Peripherally acting analgesics act by blocking the generation of impulses at chemoreceptors site of pain, while centrally acting analgesics not only raise the threshold for pain, but also alter the physiological response to pain and suppress the patient's anxiety and apprehension [27]. Abdominal writhing is associated with local peritoneal receptor. This behavior results from the activation of acidsensitive ion channels (ASICs) and transient receptor potential vanilloid-1 (TRPV1) localized in afferent primary fibers [28]. Acetic acid causes inflammatory pain by inducing capillary permeability and liberating endogenous substances that excite pain nerve ending [27], where the pain sensation is generated by producing localized inflammatory response due to release of free arachidonic acid from tissue phospholipids via cyclo-oxygenase (COX), and producing prostaglandin specifically PGE2 and PGF2 $\alpha$. The level of lipoxygenase products may also increase in peritoneal fluids [29]. More specifically, acetic acid injection induces a release of TNF- $\alpha$, interleukin- $1 \beta$ (IL-1 $\beta$ ) and interleukin-8(IL-8) by resident peritoneal macrophages, mast cells, prostanoids and bradykinin [28].These prostaglandin and lipoxygenase products are responsible for inflammation and pain. Substance(s) inhibiting the writhing response will have analgesic effect preferably by inhibition of prostaglandin synthesis, a peripheral mechanism of pain inhibition [30]. Non steroidal anti-inflammatory drugs (NSAIDs) can inhibit COX in peripheral tissues and therefore interfere with the mechanism of transduction of primary afferent nociceptors. The mechanism of analgesic activity of $D$. falcata leaves extracts could be probably due to the blockade of the effect or the release of endogenous substances that excite pain nerve endings similar to that of Morphine sulfate and NSAIDs. Thus, the statistically significant $\left(\mathrm{p}^{\mathrm{a}}<\right.$ 0.001 ) reduction in the number of writhing indicates that AEDFL, EEDFL, CEDFL and PEDFL might exert analgesic activity by inhibition of prostaglandin synthesis or by action on prostaglandin [27] (Table 1).

Tail immersion model is considered as an acute pain model. The tail-withdrawal response of mice is predominantly considered to be selective for centrally acting analgesics, implicating supraspinal analgesic pathways which is similar to the action of opioid agonists [31].The significant increase $\left(\mathrm{p}^{\mathrm{b}}<0.01, \mathrm{p}^{\mathrm{a}}<\right.$ 0.001 ) of tail-withdrawal time by the extracts suggests the involvement of central mechanisms of their analgesic effects (Table 2). Tail immersion monitors a spinal reflex involving $\mu_{2}-$ and $\delta$-opioid receptors. Therefore, the results of the study indicated that the central analgesic effect of $D$. falcata may be prominent on $\mu$ opioid receptors [31,32].

Carrageenan-induced paw edema is a convenient and well established animal model for the evaluation of anti-inflammatory effect of any natural product or synthetic chemical compound. The edema formed by carrageenan in paw has two phases. The initial phase $(1-2 \mathrm{~h})$ is predominately a non-phagocytic edema followed by a second phase $(2-5 \mathrm{~h})$ with increased edema formation that remained up to $5 \mathrm{~h}$. The inflammatory stimuli of carageenan induce the release of inflammatory mediators such as histamine, serotonin and bradykinin on vascular permeability in the initial phase. The late phase or second phase edema is attributed to the release of prostaglandin, bradykinin, protease, lysosomelike substances, growth factors and neurogenic factors [33-35]. The production of prostaglandins 
such as PGE2 and/or PGI2 is facilitated through COX-2 activity in inflammatory response [36]. As described by Loram et al. [37], inflammation induced by carrageenan involves cell migration, plasma exudation and production of mediators, such as interleukin (IL)-1 $\beta$, IL-6 and tumor necrosis factor-alpha (TNF- $\alpha$ ), a cytokineinduced neutrophil. These mediators are able to recruit leukocytes in several experimental models [38]. In addition, there are evidences showing that inflammation and/or tissue damage can induce the release of glutamate from primary afferents, enhancing the activation of excitatory amino acid receptors in the peripheral tissue. The activation of glutamate, $N$-Methyl-D-aspartate (NMDA), receptors causes the secretion of inflammatory mediators such as TNF-a and IL-1b that in turn can sensitize peripheral nerve endings and activate the immune response [11]. Recently, it has also been reported that NO was produced by isoform of nitric oxide synthase (iNOS) which is involved in the inflammatory response on paw edema. The anti-inflammatory drugs such as aspirin and ibuprofan have been found to inhibit prostaglandin production that is the main reason of anti-inflammatory action in carrageenaninduced paw edema [36,39]. All the extracts (AEDFL, EEDFL, CEDFL and PEDFL) significantly $\left(\mathrm{p}^{\mathrm{b}}<0.01, \mathrm{p}^{\mathrm{a}}<0.001\right)$ reduced paw edema volume in dose dependant manner (Table 3). From the results, it is suggested that the antiinflammatory effect of the extracts on carrageenan-induced paw edema may act via the inhibition of NO production, along with the inhibitory activity of COX-2 [36].

CNS depressant effect of $D$. falcata leaves was studied using two neuropharmacological models (Open field and Hole cross). The results of the study provided evidence that the extracts reduced locomotor activity confirming its CNS depressant potential. Locomotor activity is considered as an index of alertness and a reduction of it is an indicative of sedative or CNS depressant activity [40]. Gamma-aminobutyric acid (GABA) is the major inhibitory neurotransmitter in the central nervous system [41], which is also involved in other physiological functions related to behavior and in various psychological and neurological disorders such as epilepsy, anxiety, depression, parkinson syndrome, and alzheimer's disease [42]. Diverse drugs such as anxiolytic, muscle relaxant and sedative-hypnotic exhibit their action via GABA. These drugs might modify the GABA system, induce anxiolysis or hypnosis in animals, at the level of the synthesis of it by potentiating the GABA-mediated postsynaptic inhibition through an allosteric modification of GABA receptors, and by direct increase in chloride conductance or indirectly by potentiating GABA-induced chloride conductance with simultaneous depression of voltage activated $\mathrm{Ca}^{++}$currents like barbiturates [43].Therefore, it is predictable that extracts of $D$. falcata leaves may act by potentiating GABAergic inhibition in the CNS via membrane hyper-polarization leading to a reduction in the firing rate of critical neurons in the brain or it may be due to direct activation of GABA receptors by the extracts [41]. It may also be due to enhanced affinity for GABA or an increase in the duration of the GABA-gated channel opening [25]. In addition, the study on locomotor activity, as measured by hole cross and open field tests, showed that all doses of the extracts significantly $\left(\mathrm{p}^{\mathrm{b}}<0.01, \mathrm{p}^{\mathrm{a}}<0.001\right)$ decreased the frequency and the amplitude of movements in dose dependant manner from 2nd observation (30 min) and continued up to the 5th observation (120 min) period (Table 4 and 5). Since the locomotor activity is a measure of the level of excitability of the CNS, this decrease in spontaneous motor activity could be attributed to the depressant effect of the plant extracts with the presence of compound(s) having CNS depressant potential [44].

It is well established that alkaloids, flavonoids and tannins are potent analgesic compounds [45]. Flavanoids exert their effect through inhibition of prostaglandin synthatase [46]. Daniela T. et al. showed the analgesic potential of a triterpene by inhibiting acetic acidinduced viscerosomatic nociception and cellular migration. In addition, it exhibited antiinflammatory effect by reducing leukocyte migration and carrageenan-induced cytokine release, namely tumor necrosis factor a (TNF-a) and interleukin-1b (IL-1b) [11]. Various flavonoid derivatives including quercetin have 
been reported to inhibit the activity of arachidonic acid metabolizing enzymes (phospholipase A2, cycloxygenase and lipoxygenase), and some flavonoids have proved to exert anti-inflammatory actions by modulation of proinflammatory gene expression, such as COX-2, i-NOS and several pivotal cytokines [36]. Steroidal saponins are significant inhibitor of inflammatory cytokines by activated macrophages [47]. Oxyresveratrol a potent antioxidant has been reported to exert antiinflammatory activity through inhibition of iNOS/NO production, PGE2 synthesis and NFאB activation [48]. Epicathechin and (+) catechins are reported to have many biological effects including anti-inflammatory response [33]. Flavone and isoflavone suppresses inflammatory response by inhibiting NF- $\mathrm{BB}$ [49]. Control of different cellular processes such as inflammation or apoptosis are modulated by polyphenols [50]. Tannins are known to be useful for the prevention of cancer as well as treatment of inflamed or ulcerated tissues [45].

Research has shown that plants containing flavonoids, saponins and tannins are useful for the treatment of many CNS disorders [40]. Earlier investigation of the phytoconstituents of medicinal plants suggests that many flavonoids and neuroactive steroids are ligands for $\mathrm{GABA}_{\mathrm{A}}$ receptors in the central nervous system which suggests that they can act as benzodiazepine-like agents [40]. It has also been reported that some flavanoids exhibit high affinity binding to the benzodiazepine site of $\mathrm{GABA}_{\mathrm{A}}$ receptors. Therefore, the CNS-depressant activity may be due to the phytoconstituents present in the different extracts of the $D$. falcata leaves. Raihan M.O. et al. reported that $\beta$-sitosterol act as $\mathrm{GABA}_{\mathrm{A}}$ agonists [51]. Several plants have been reported to have CNS depressant activity due to the presence of triterpenoids, saponins and flavonoids. Triterpenoid saponins are reported to have agonistic/facilitatory activities at $\mathrm{GABA}_{\mathrm{A}}$ receptor complex $[43,44,52]$. This is supported by their behavioral effects in animal models of CNS depression and anxiety.

Literature review revealed the presence of tannins, flavonoids, phenolic compounds, phytosterols, triterpenes, quercetin, $\beta$-sitosterol, triterpenes etc. bioactive compounds in the extracts of $D$. falcata leaves which are believed to be responsible for analgesic, anti-inflammatory and CNS-depressant effects of the plant. There is no strict evidence about which substances are exactly responsible for these effects. However, further studies are necessary to find out the key compound(s) responsible for these effects, and to identify the mechanism of action and the pharmacodynamics of these effects. More research and clinical trials could help to identify agents with more potent CNS depressant effects.

\section{Conclusion}

In conclusion, the results presented in this study revealed that crude ethanol extract and aqueous, chloroform as well as petroleum ether fractions of $D$. falcate (Linn.) leaves possess significant analgesic, anti-inflammatory and CNS depressant potentials. Petroleum ether (PEDFL) and ethanol extracts (EEDFL) have shown highly significant activities in mice than chloroform (CEDFL) and aqueous (AEDFL) extracts. These results further support the traditional use of this plant in medicine. The potential of the extracts as analgesic, anti-inflammatory and CNS depressant activities may be due to the presence of phytoconstituents like flavonoids, tannins, phenolics etc. However, more detail phytochemical analysis is necessary to isolate and characterize the active compounds which are responsible for these activities, and that will give a way to draw the proper mechanisms of action of these activities.

\section{Acknowledgments}

The authors are grateful to Pharmacy department of Southeast University, Dhaka, Bangladesh for providing all required facilities to conduct the research in their lab. The authors are also thankful to animal division of the International Centre for Diarrhoeal Disease and Research, Bangladesh (ICDDR, B) for timely providing sufficient experimental mice.

Conflict of interest: Authors have declared that they have no conflict of interest. 


\section{References}

1. Pooja, S.; Irchhaiya, R.; Bhawna, S.; Gayatri, S.; Santosh, K. Anticonvulsant and muscle relaxant activity of the ethanolic extract of stems of Dendrophthoe falcata (Linn. F.) in mice, Indian Journal of Pharmacology, 2011, 43(6), 710-713.

[DOI: 10.4103/02537613.89832];

[PubMed: 22144780].

2. Pattanayak, S.P.; Mazumder, P.M. Immunomodulatory activities of Dendrophthoe falcata (L.f) Ettingsh in experimental animals: In vitro and In vivo investigations, Journal of Scientific Research, 2011, 3 (3), 619-630. [DOI:10.3329/jsr.v3i3.7655].

3. Nipun, D.; Vijay, S.; Kirti, S.; Prabhu; Richard, L. In vitro cytotoxic activity of Dendrophthoe falcata on human breast adenocarcinoma cells-MCF-7, International Journal of Cancer Research, 2011, 7(1), 4754. [DOI: 10.3923/ijcr.2011.47.54].

4. Satish, P.; Sneha, A.; Ram, J.; Sanjay, S. Evaluation of anti-inflammatory activity and in-vitro antioxidant activity of Indian mistletoe, the hemiparasite Dendrophthoe falcata L. F. (Loranthaceae), Iranian Journal of Pharmaceutical Research, 2011, 10(2), 253-259. [PubMed: 24250351].

5. Karthikeyan, A.; Rameshkumar, R.; Sivakumar, N.; Al-Amri, I.S.; Karutha, P. S.; Ramesh, M. Antibiofilm activity of Dendrophthoe falcata against different bacterial pathogens, Planta Medica, 2012, 78(18), 1918-1926. [DOI: 10.1055/s-00321327879]; [PubMed:23115018]

6. Pattanayak, S.P.; Mazumder, P.M. Contraception effect of Dendrophthoe falcata (L.f.) Ettingsh on female reproductive system in Wistar rats: a focus on antifertility efficacy, Contraception, 2009, 80(3), 314-322. [DOI: 10.1016/j.contraception.2009.03.001];

[PubMed: 19698827].
7. Gupta, R.S.; Kachhawa, J.B.; Sharma, A. Effect of methanolic extract of Dendrophthoe falcata stem on reproductive function of male albino rats, Journal of Herbal Pharmacotherapy, 2007, 7(2), 1-13. DOI:10.1300/J157v07n02_01;

[PubMed:18285304].

8. Sathishkumar, G.; Gobinath, C.; Wilson, A.; Sivaramakrishnan, S. Dendrophthoe falcata (L.f) Ettingsh (Neem mistletoe): A potent bioresource to fabricate silver nanoparticles for anticancer effect against human breast cancer cells (MCF-7), Spectrochimca Acta part A: Molicular and Biomolicular Spectroscopy, 2014, 7(128), 285-290. [DOI: 10.1016/j.saa.2014.02.096].

9. Simeon, K. A.; Illoh, H.C.; Imoh I. J.; Imo E.J. African mistletoes (Loranthaceae); Ethnopharmacology, chemistry and medicinal values: an update, African Journal of Traditional, Complementary and Alternative Medicines, 2013, 10(4), 161-170. [http://www.ncbi.nlm.nih.gov/pmc/articles/P MC3794408/pdf/AJT1004-0161.pdf];

[PubMed: 24146518].

10. Uppuluri, V.M.; Kilambi, N.; Akella V.S.S.; Anita, M.; Wenkui, L.; Richard, B.V. Three new pentacyclc triterpenes and some flavanoids from the fruits of Indian ayurvedic plant Dendrophthoe falcata and their receptor binding activity, Chemical and Pharmacetical Bulletin 2006, 54, 740 - 744. [DOI:10.1002/chin.200644181]; [PubMed: 16651782].

11. Daniela, T.L.; De 'bora, L.; Cristiane, H.B.; Morgana, D.S.; Celeste, H.C.; Valdir, A.F.; Adair, R.S.S. Anti-inflammatory effect of triterpene 3b,6b,16b-trihydroxylup-20(29)ene obtained from Combretum leprosum Mart \& Eich in mice, Journal of Ethnopharmacology, 2012, 142, 59-64. [DOI: 10.1016/j.jep.2012.04.013]. [PubMed: 22575213].

12. Xueli, Z.; Yang, Y.; Zhou, C.; Shan, L.; Yunguo, Z.; Xiaoling, Y. Anti-depressant effect of Chimonanthus salicifolicus essential oil in chronic stressed rats, Journal of Medicinal Plant Research, 2014, 8(10), 430435. [DOI: 10.5897/JMPR11.1709]. 
13. Raman, C.; Pallavi, T.; Ayush, C.; Sarita, J.; Anamika, S.; Rajeev, G.; Jyoti, S.; Smruti, S. P.; Vinod, K.; Rakesh, K.S.; Rajesh, A. Evidence based herbal drug standardization approach in coping with challenges of holistic management of diabetes: a dreadful lifestyle disorder of 21st century, Journal of Diabetes \& Metabolic Disorders, 2013, 12, 35. [DOI: 10.1186/2251-6581-12-35]; PubMed: 23822656].

14. Prabhjit, K.; Satwinderjeet, K.; Subodh, K.; Palwinder, S. Rubia cordifolia L. and Glycyrrhiza glabra L. medicinal plants as potential source of $\mathrm{COX}-2$ inhibitors, American Journal of Biomedical Sciences, 2010, 2(2),108-120. [DOI: 10.5099/aj100200108].

15. Jiajiu, S.; Ben, Chen.; Paul, W.; Wen-Hsin, H.; An-Rong, L.; Dustin, Z. Antiinflammatory and anti-arthritic effects of a novel leflunomide analogue, UTL-5b (GBL5b), American Journal of Biomedical Sciences, 2011, 3(1), 31-39. [DOI: 10.5099/aj110100031]; [PubMed: 21253441].

16. Dayang, E. Z.; Awang, H.; Roslida, A.H.; Latifah, S.Y.; Che, N.M.T.; Yeong, L. T. The hexane fraction of Ardisia crispa Thunb. A. DC. roots inhibits inflammation-induced angiogenesis. BMC Complementary and Alternative Medicine 2013, 13, 5. [DOI: 10.1186/1472-6882-13-5]; $\quad$ [PubMed: 23298265].

17. Hwan-Suck, C.; Hye, J.L.; Insop, S.; Hyunsu, B. Assessment of anti-depressant effect of nelubinis- semen on rats under chronic mild stress and its subchronic oral toxicity in rats and beagle dogs, BMC Complementary and Alternative Medicine, 2012, 12, 68. [DOI: 10.1186/1472-6882-12-68]; [PubMed: 22640371].

18. Kim, J.H.; Chung, H.S.; Kang, M.; Kim, Y.; Kim, B.S.; Kim, Y.S.; Bae, H. Anti-diabetic effect of standardized herbal formula PM021 consisting of Mori folium and Aurantii fructus on type IIdiabetic Otsuka Long-Evans tokushima fatty (OLETF) rats, Diabetes Research and Clinical Practice, 2011, 93(2), 198-204.

[DOI: 10.1016/j.diabres.2011.03.037]; [PubMed:

21524812].

19. Zimmermann, M. Ethical guidelines for investigations of experimental pain in concious animals, Pain, 1983, 16, 109-110.

20. Koster, R.; Anderson, M.; Debeer, E. J. Acetic acid for analgesic screening, Federation Proceedings, 1959, 18, 412-416.

21. Toma, W.; Graciosa, J.S.; Hiruma-Lima, C.A.; Andrade, F.D.P.; Vilegas, W.; Souza, B. A.R.M. Evaluation of the analgesic and antiedematogenic activities of Quassia amara bark extract, Journal of Ethnopharmacology, 2003, 85, 19-23. [DOI:10.1016/S03788741(02)00334-3]; [PubMed:12576198].

22. Tan-no, K.; Nakajima, T.; Shoji, T.; Nakagawasai, O.; Niijima, F.; Ishikawa, M.; Endo, Y.; Sato, T.; Satoh, S.; Tadano, T. Anti-inflammatory effect of propolis through inhibition of nitric oxide production on carrageenin-induced mouse paw edema, Biological and Pharmaceutical Bulletin, 2006, 29, 96-99. [DOI:10.1248/bpb.29.96]; [PubMed:16394518].

23. Gupta, B. D.; Dandiya, P. C.; Gupta, M. L. A psycho-pharmacological analysis of behaviour in rats, The Japanese Journal of Pharmacology, 1971, 21, 293-298. [DOI: 10.1254/jip.21.293] [PubMed: 4397666].

24. Takagi, K.; Watanabe, M.; Saito, H. Studies of the spontaneous movement of animals by the hole cross test; effect of 2-dimethylaminoethanol and its acyl esters on the central nervous system, The Japanese Journal of Pharmacology, 1971, 21, 797-810. [DOI:10.1254/jip.21.797]; [PubMed: 5316865].

25. Shans-Ud-Doha, K.M.; Zobaer, A. M.; Sitesh, C. B.; Nazmul, Q. Antinociceptive, antiinflammatory, antimicrobial and central nervous system depressant activities of ethanolic extract of leaves and roots of Gomphostemma parviflorum var. parviflorum wall, Pharmacognosy Research, 2013, 5(4), 233-240. [DOI: 10.4103/0974-8490.118777]; [PubMed: 24174815].

26. Marrassini, C.; Acevedo, C.; Miño, J.; Ferraro, G.; Gorzalczany, S. Evaluation of antinociceptive, anti-inflammatory activities 
and phytochemical analysis of aerial parts of Urtica urens L., Phytotherapy Research, 2010, 24, 1807-1812. [DOI: 10.1002/ptr.3188]; [PubMed: 20564509].

27. Chandana, C.B.; Jayanti, D.R.; Bhaben, B.; Acheenta, G.B.; Prabodh, B.; Mangala, L. Analgesic and anti-nociceptive activity of hydroethanolic extract of Drymaria cordata willd, Indian Journal of Pharmacology, 2011, 43(2), 121-125. [DOI: 10.4103/02537613.77337]; [PubMed: 21572643].

28. Luiz, H.A. C.; Brito da Matta, C.B; de Araújo, M. V.; Barbosa-Filho, J. M.; de Lira, D. P.; de Oliveira Santos, B. V. Antinociceptive and anti-inflammatory activities of crude methanolic extract of red alga Bryothamnion triquetrum, Marine Drugs, 2012, 10, 1977-1992. [DOI: 10.3390/md10091977]; [PubMed: 23118715].

29. Haroon, K.; Muhammad, S.; Anwal-UlHassan, G.; Murad, A. K.; Ahsana, D.; Inamullah, K. The antinociceptive activity of Polygonatum verticillatum rhizomes in pain models, Journal of Ethnopharmacology, 2010, 127(2), 521-527. [DOI: 10.1016/j.jep.2009.10.003]; [PubMed: 19853648].

30. Pedro, G.S.; Xirley, P.N.; Julianeli, T.L.; José, A.S. F.; André, P. F.; Jullyana S.S.; Lucindo, J.Q.; Patrícia, K. F. D.; Carla R.C.B.; Alexsandro, B.; Jackson, R.G.S.A. Antinociceptive effect of ethanolic extract of Selaginella convoluta in mice, BMC Complementary and Alternative Medicine, 2012, 12, 187. [DOI: 10.1186/1472-6882-12187]; [PubMed:23082856].

31. Mohammad, Z.I.; Chandra, D. S. Evaluation of antinociceptive activity of hydromethanol extract of Cyperus rotundus in mice, BMC Complementary and Alternative Medicine, 2014, 14, 83. [DOI: 10.1186/1472-6882-1483]; [PubMed: 24589067].

32. Arslan, R.; Bektas, N. Antinociceptive effect of methanol extract of Capparis ovata in mice, Pharmaceutical Biology, 2010, 48, 1185-1190.

[DOI: 10.3109/13880201003629323]; [PubMed: 20819022].
33. Carina, D.; Priscilla, S.M.; Marcelo, F.; Adna, P. M.; Keityane, B. B.; Severino, M. A.; Pedro, L.R. Guava pomace: a new source of anti-inflammatory and analgesic bioactives, BMC Complementary and Alternative Medicine, 2013, 13, 235. [DOI: 10.1186/1472-6882-13-235]; [PubMed: 24063346].

34. Naveed, M.; Muhammad, S.; Haroon, K. Antipyretic, analgesic and anti-inflammatory activity of Viola betonicifolia whole plant, BMC Complementary and Alternative Medicine, 2012, 12, 59. [DOI: 10.1186/14726882-12-59]; [PubMed: 22551220].

35. Yeping, R.; Li, Y.; Bingbing, Z.; Shuijuan, Z.; Jianyou, G. Anti-inflammatory effects of Neurotoxin-Nna, a peptide separated from the venom of Naja naja atra, BMC Complementary and Alternative Medicine, 2013, 13, 86. [DOI: 10.1186/1472-6882-1386]; [PubMed: 23587180].

36. Hiroe, M.; Takashi, S.; Yoko, A.; Hideaki, H. Anti-inflammatory effect of bee pollen ethanol extract from Cistus sp. of Spanish on carrageenan-induced rat hind paw edema, BMC Complementary and Alternative Medicine, 2010, 10, 30. [DOI: 10.1186/14726882-10-30]; [PubMed: 20573205].

37. Makson, G.B.O.; Rosemarie, B.M.; Michele, F. S.; Amanda, B.D.S.; Fabíola, A.B.; Emiliano, O.B.; Damião, P.D.S.; Fernanda, R.C.A.; Daniel, B.J.; Ângelo, R.A.; Lucindo, J.Q. $\alpha$-terpineol reduces mechanical hypernociception and inflammatory response, Basic \& Clinical Pharmacology \& Toxicology, 2012, 111(2), 120-125. [DOI: 10.1111/j.1742-7843.2012.00875.x];

[PubMed: 22380944].

38. Claudio, M. L.; Adriana, K.L.; Marcelia, G.D.M.; Mairim, R.S.; Dênisson, L.O.; Enrik, B.A.; Rosana, S.S. B.; Paulo, C. L.N.; Valéria, R.S.M.; Édica, R. A.O.; Ricardo, L.C. A.; Lucindo, J.Q.; Adriano, A.S.A. Bioassay-guided evaluation of Dioscorea villosa-an acute and subchronic toxicity, antinociceptive and anti-inflammatory approach, BMC Complementary and Alternative Medicine, 2013, 13, 195. [DOI: 
10.1186/1472-6882-13-195]; $\quad$ [PubMed: 23889998].

39. Lee, K.P.; Sudjarwo, G.W.; Kim, J.S.; Dirgantara, S.; Maeng, W.J.; Hong, H. The anti-inflammatory effect of Indonesian Areca catechu leaf extract in vitro and in vivo, Nutrition Research Practice, 2014, 8(3), 267271. [DOI: $10.4162 / \mathrm{nrp} .2014 .8 .3 .267] ;$ [PubMed: 24944770].

40. Protapaditya, D.; Sangita, C.; Priyanka, C.; Sanjib, B. Neuropharmacological properties of Mikania scandens (L.) Willd. (Asteraceae), Journal of Advanced Pharmaceutical Technology and Research, 2011, 2(4), 255259.[DOI: 10.4103/2231-4040.90883];

[PubMed: 22247893].

41. Kavita, G.; Vijay, K. L.; Shivesh, J. Anticonvulsant potential of ethanol extracts and their solvent partitioned fractions from Flemingia strobilifera root, Pharmacognosy Research, 2013, 5(4), 265-270. [DOI: 10.4103/0974-8490.118825]; [PubMed: 22979938].

42. Kumar, K.; Sharma, S.; Kumar, P.; Deshmukh, R. Therapeutic potential of $\mathrm{GABA}(\mathrm{B})$ receptor ligands in drug addiction, anxiety, depression and other CNS disorders, Pharmacology Biochemistry and Behavior, 2013 ;110:174-184. [DOI: 10.1016/j.pbb.2013.07.003]; [PubMed: 2387236].

43. Uma, A.B.; Radha, Y.; Prachi, D.P.; Mandar, R.Z.; Rahul, S.S. Study of central nervous system depressant and behavioral activity of an ethanol extract of Achyranthes aspera (Agadha) in different animal models, International Journal of Applied and Basic Medical Research, 2011, 1(2), 104-108. [DOI: 10.4103/2229-516X.91154]; [PubMed: 23776787].

44. Kumaresan, P.T.; Asish, T.; Vijaya, C. Neuropharmacological activity of Lippia nodiflora Linn, Pharmacognosy Research, 2011, 3(3), 194-200. [DOI: 10.4103/09748490.85007]; [PubMed: 22022169].
45. Olubunmi, A.W.; Anthony, J.A. Phytochemical constituents and antioxidant activities of the whole leaf extract of Aloe ferox Mill, Pharmacognosy Magazine, 2011, 7(28), 325-333. [DOI: 10.4103/09731296.90414]; [PubMed: 3261067].

46. Mojahid-ul-Islam; Sanadelaslam E. Phytochemical investigation and evaluation of analgesic activity of ethonolic extract of Dalbergia sissoo (Roxb.) bark, Journal of Natural Product and Plant Resources, 2012, 2(6), 701-704. [DOI: 10.4103/09731296.90414]; [PubMed:22262936].

47. Camila, R.A.; Bernadete, P.S.; José, P.P. A new steroidal saponin with antiinflammatory and antiulcerogenic properties from the bulbs of Allium ampeloprasum var. porrum, Fitoterapia, 2011, 82, 1175-1180. [DOI: 10.1016/j.fitote.2011.08.003]; [PubMed: 21856386].

48. Yi-Ching, C.; Yin-Jing, T.; Chun-Houh, C.; Francesca, N. B.; Evangeline, C.A.; Ran-Juh, W.; Den-Jen, W.; Clément M.; Yea-Lih, L.W.Y. Morus alba and active compound oxyresveratrol exert anti-inflammatory activity via inhibition of leukocyte migration involving MEK/ERK signaling. $B M C$ Complementary and Alternative Medicine, 2013, 13, 45. [ DOI: 10.1186/1472-6882-1345]; [PubMed: 23433072].

49. Juyeong, L.; Gabsik, Y.; Kyungjin, L.; MiHwa, L.; Ji-Whan, E.; Inhye, H.; Ho-Young, C. Anti-inflammatory effect of Prunus yedoensis through inhibition of nuclear factor- $\mathrm{KB}$ in macrophages, $B M C$ Complementary and Alternative Medicine, 2013, 13, 92. [DOI: 10.1186/1472-6882-1392]; [PubMed: 23631356].

50. Praveen, N.; Anshoo, M.; Devinder, K. D. $\mathrm{COX}-2$ as a potential target in chemoprevention of Benzo(a) pyrene induced lung carcinogenesis in mice-combined role of curcumin and quercetin, American Journal of Biomedical Sciences, 2012, 4(3), 194-203. [DOI: 10.5099/aj120300194].

51. Raihan, M.O.; Habib, M.R.; Afrina, B.; Mominur, R. M.M.; Moshfiqus, S. M.M.; Mashudul M. Sedative and anxiolytic effects of the methanolic extract of Leea indica 
(Burm. f.) Merr. Leaf, Drug Discoveries \& Therapeutics, 2011, 5(4), 185-189. [DOI:10.5582/ddt.2011.v5 .4.185].

52. Khatoon, M.M.; Khatun, M.H.; Islam, M.E.; Parvin, M.S. Analgesic, antibacterial and central nervous system depressant activities of Albizia procera leaves, Asian Pacific
Journal of Tropical Biomedicine, 2014, 4(4), 279-284.

[DOI:10.12980/APJTB.4.2014C348]. 